\title{
ON A FLOW OF TRANSFORMATIONS OF A WIENER SPACE
}

\author{
J. NAJNUDEL, D. STROOCK, M. YOR
}

\begin{abstract}
In this paper, we define, via Fourier transform, an ergodic flow of transformations of a Wiener space which preserves the law of the Ornstein-Uhlenbeck process and which interpolates the iterations of a transformation previously defined by Jeulin and Yor. Then, we give a more explicit expression for this flow, and we construct from it a continuous gaussian process indexed by $\mathbb{R}^{2}$, such that all its restriction obtained by fixing the first coordinate are Ornstein-Uhlenbeck processes.
\end{abstract}

\section{INTRODUCTION}

An abstract Wiener space is a triple $(H, E, \mathcal{W})$ consisting of a separable, real Hilbert space $H$, a separable real Banach space $E$ in which $H$ is continuously embedded as a dense subspace, and a Borel probability measure $\mathcal{W}$ on $E$ with the property that, for each $x^{*} \in E^{*}$, the $\mathcal{W}$-distribution of the map $x \in E \longmapsto\left\langle x, x^{*}\right\rangle \in \mathbb{R}$, from $E$ to $\mathbb{R}$, is a centered gaussian random distribution with variance $\left\|h_{x^{*}}\right\|_{H}^{2}$, where $h_{x^{*}}$ is the element of $H$ determined by $\left(h, h_{x^{*}}\right)_{H}=\left\langle h, x^{*}\right\rangle$ for all $h \in H$. See Chapter 8 of [5] for more information on this topic.

Because $\left\{h_{x^{*}}: x^{*} \in E^{*}\right\}$ is dense in $H$ and $\left\|h_{x^{*}}\right\|_{H}=\left\|\left\langle\cdot, x^{*}\right\rangle\right\|_{L^{2}(\mathcal{W})}$, there is a unique isometry, known as the Paley-Wiener map, $\mathcal{I}: H \longmapsto L^{2}(\mathcal{W})$ such that $\mathcal{I}(h)=\left\langle\cdot, x^{*}\right\rangle$ if $h=h_{x^{*}}$. In fact, for each $h \in H, \mathcal{I}(h)$ under $\mathcal{W}$ is a centered Gaussian variable with variance $\|h\|_{H}^{2}$. Because, when $h=h_{x^{*}}, \mathcal{I}(h)$ provides an extention of $(\cdot, h)_{H}$ to $E$, for intuitive purposes one can think of $x \rightsquigarrow[\mathcal{I}(h)](x)$ as a giving meaning to the inner product $x \rightsquigarrow(x, h)_{H}$, although for general $h$ this will be defined only up to a set of $\mathcal{W}$-measure 0 .

An important property of abstract Wiener spaces is that they are invariant under orthogonal transformations on $H$. To be precise, given an orthogonal transformation $\mathcal{O}$ on $H$, there is a $\mathcal{W}$-almost surely unique $T_{\mathcal{O}}: E \longrightarrow E$ with the property that, for each $h \in H$, $\mathcal{I}(h) \circ T_{\mathcal{O}}=\mathcal{I}\left(\mathcal{O}^{\top} h\right) \mathcal{W}$-almost surely. Notice that this is the relation which one would predict if one thinks of $[\mathcal{I}(h)](x)$ as the inner product of $x$ with $h$. In general, $T_{\mathcal{O}}$ can be constructed by choosing $\left\{x_{m}^{*}: m \geq 1\right\} \subseteq E^{*}$ so the $\left\{h_{x_{m}^{*}}: m \geq 1\right\}$ is an orthonormal basis in $H$ and then taking

$$
T_{\mathcal{O}} x=\sum_{m=1}^{\infty}\left\langle x, x_{m}^{*}\right\rangle \mathcal{O} h_{x_{m}^{*}},
$$

where the series converges in $E$ for $\mathcal{W}$-almost every $x$ as well as in $L^{p}(\mathcal{W} ; E)$ for every $p \in[1, \infty)$. See Theorem 8.3.14 in [5] for details. In the case when $\mathcal{O}$ admits an extension as a continuous map on $E$ into itself, $T_{\mathcal{O}}$ can be the taken equal to that extension. In any case, it is an easy matter to check that the measure $\mathcal{W}$ is preserved by $T_{\mathcal{O}}$. Less obvious is a theorem, originally formulated by I.M. Segal (cf. [6]), which says that $T_{\mathcal{O}}$ is ergodic if and only $\mathcal{O}$ admits no non-trivial, finite dimensional, invariant subspace. Equivalently, $T_{\mathcal{O}}$

Date: April 20, 2022. 
is ergodic if and only if the complexification $\mathcal{O}_{\mathrm{c}}$ has a continuous spectrum as a unitary operator on the complexification $H_{\mathrm{c}}$ of $H$.

The classical Wiener space provides a rich source of examples to which the preceding applies. Namely, take $H=H_{0}^{1}$ to be the space of absolutely continuous $h \in \Theta$ whose derivative $\dot{h}$ is in $L^{2}([0, \infty))$, and set $\|h\|_{H_{0}^{1}}=\|\dot{h}\|_{L^{2}([0, \infty))}$. Then $H_{0}^{1}$ with norm $\|\cdot\|_{H_{0}^{1}}$ is a separable Hilbert space. Next, take $E=\Theta$, where $\Theta$ is the space of continuous paths $\theta:[0, \infty) \longrightarrow \mathbb{R}$ such that $\theta(0)=0$ and

$$
\frac{|\theta(t)|}{t^{\frac{1}{2}} \log (e+|\log t|)} \longrightarrow 0 \text { as } t>0 \text { tends to } 0 \text { or } \infty,
$$

and set

$$
\|\theta\|_{\Theta}=\sup _{t>0} \frac{|\theta(t)|}{t^{\frac{1}{2}} \log (e+|\log t|)} .
$$

Then $\Theta$ with norm $\|\cdot\|_{\Theta}$ is a separable Banach space in which $H_{0}^{1}$ is continuously embedded as a dense subspace. Finally, the renowned theorem of Wiener combined with the Brownian law of the iterated logarithm says that there is a Borel probability measure $\mathcal{W}_{H_{0}^{1}}$ on $\Theta$ for which $\left(H_{0}^{1}, \Theta, \mathcal{W}_{H_{0}^{1}}\right)$ is an abstract Wiener space. Indeed, it is the classical Wiener space on which the abstraction is modeled, and $\mathcal{W}_{H_{0}^{1}}$ is the distribution of an $\mathbb{R}$-valued Brownian motion.

One of the simplest examples of an orthogonal transformation on $H_{0}^{1}$ for which the associated transformation on $\Theta$ is ergodic is the Brownian scaling map $S_{\alpha}$ given by $S_{\alpha} \theta(t)=$ $\alpha^{-\frac{1}{2}} \theta(\alpha t)$ for $\alpha>0$. It is an easy matter to check that the restriction $\mathcal{O}_{\alpha}$ of $S_{\alpha}$ to $H_{0}^{1}$ is orthogonal, and so, since $S_{\alpha}$ is continuous on $\Theta$, we can take $T_{\mathcal{O}_{\alpha}}=S_{\alpha}$. Furthermore, as long as $\alpha \neq 1$, an elementary computation shows that $\lim _{n \rightarrow \infty}\left(g, \mathcal{O}_{\alpha}^{n} h\right)_{H}=0$, first for smooth $g, h \in H_{0}^{1}$ with compact support in $(0, \infty)$ and thence for all $g, h \in H_{0}^{1}$. Hence, when $\alpha \neq 1$, $\mathcal{O}_{\alpha}$ admits no non-trivial, finite dimensional subspace, and therefore $S_{\alpha}$ is ergodic; and so, by the Birkoff's Individual Ergodic Theorem, for $p \in[1, \infty)$ and $f \in L^{p}\left(\mathcal{W}_{H_{0}^{1}}\right)$,

$$
\lim _{n \rightarrow \infty} \frac{1}{n} \sum_{m=0}^{n-1} f \circ S_{\alpha}^{n}=\int f d \mathcal{W}_{H_{0}^{1}}
$$

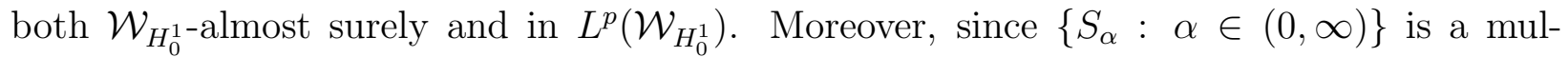
tiplicative semigroup in the sense that $S_{\alpha \beta}=S_{\alpha} \circ S_{\beta}$, one has the continuous parameter version

$$
\lim _{a \rightarrow \infty} \frac{1}{\log a} \int_{1}^{a}\left(f \circ S_{\alpha}\right) \frac{d \alpha}{\alpha}=\int f d \mathcal{W}_{H_{0}^{1}}
$$

of the preceding result.

A more challenging ergodic transformation of the classical Wiener space was studied by Jeulin and Yor (see [1], [2] and [4]), and, in the framework of this article, it is obtained by considering the transformation $\mathcal{O}$ on $H_{0}^{1}$, defined by

$$
[\mathcal{O} h](t)=h(t)-\int_{0}^{t} \frac{h(s)}{s} d s
$$


An elementary calculation shows that $\mathcal{O}$ is orthogonal. Moreover, $\mathcal{O}$ admits a continuous extension to $\Theta$ given by replacing $h \in H_{0}^{1}$ in (1.1) by $\theta \in \Theta$. That is

$$
\left[T_{\mathcal{O}} \theta\right]=\theta(t)-\int_{0}^{t} \frac{\theta(s)}{s} d s \quad \text { for } \theta \in \Theta \text { and } t \geq 0 .
$$

In addition, one can check that $\lim _{n \rightarrow \infty}\left(g, \mathcal{O}^{n} h\right)_{H_{0}^{1}}=0$ for all $g, h \in H_{0}^{1}$, which proves that $T_{\mathcal{O}}$ is ergodic for $\mathcal{W}_{H_{0}^{1}}$.

In order to study the transformation $T_{\mathcal{O}}$ in greater detail, it will be convenient to reformulate it in terms of the Ornstein-Uhlenbeck process. That is, take $H^{U}$ to be the space of absolutely continuous functions $h: \mathbb{R} \longrightarrow \mathbb{R}$ such that

$$
\|h\|_{H^{U}} \equiv \sqrt{\int_{\mathbb{R}}\left(\frac{1}{4} h(t)^{2}+\dot{h}(t)^{2}\right) d t}<\infty .
$$

Then $H^{U}$ becomes a separable Hilbert space with norm $\|\cdot\|_{H^{U}}$. Moreover, the map $F$ : $H_{0}^{1} \longrightarrow H^{U}$ given by

$$
[F(g)](t)=e^{-\frac{t}{2}} g\left(e^{t}\right), \quad \text { for } g \in H_{0}^{1} \text { and } t \in \mathbb{R},
$$

is an isometric surjection which extends as an isometry from $\Theta$ onto Banach space $\mathcal{U}$ of continuous $\omega: \longrightarrow \mathbb{R}$ satisfying $\lim _{|t| \rightarrow \infty} \frac{|\omega(t)|}{\log |t|}=0$ with norm $\|\omega\|_{\mathcal{U}}=\sup _{t \in \mathbb{R}}(\log (e+|t|))^{-1}|\omega(t)|$. Thus, $\left(H^{U}, \mathcal{U}, \mathcal{W}_{H^{U}}\right)$ is an abstract Wiener space, where $\mathcal{W}_{H^{U}}=F_{*} \mathcal{W}_{H_{0}^{1}}$ is the image of $\mathcal{W}_{H_{0}^{1}}$ under the map $F$. In fact, $\mathcal{W}_{H^{U}}$ is the distribution of a standard, reverisible OrnsteinUhlenbeck process.

Note that the scaling transformations for the classical Wiener space become translations in the Ornstein-Uhlenbeck setting. Namely, for each $\alpha>0, F \circ S_{\alpha}=\tau_{\log \alpha} \circ F$, where $\tau_{s}$ denotes the time-translation map given by $\left[\tau_{s} \omega\right](t)=\omega(s+t)$. Thus, for $s \neq 0$, the results proved about the scaling maps say that $\tau_{s}$ is an ergodic transformation for $\mathcal{W}_{H^{U}}$. In particular, for $p \in[1, \infty)$ and $f \in L^{p}\left(\mathcal{W}_{H^{U}}\right)$,

$$
\lim _{n \rightarrow \infty} \frac{1}{n} \sum_{m=0}^{n-1} f \circ \tau_{n s}=\lim _{T \rightarrow \infty} \frac{1}{T} \int_{0}^{T} f \circ \tau_{s} d s=\int f d \mathcal{W}_{H^{U}}
$$

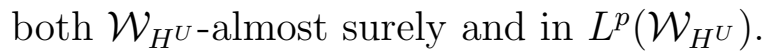

The main goal of this article is to show that the reformulation of transformation $T_{\mathcal{O}}$ coming from the Jeulin-Yor transformation in terms of the Ornstein-Uhlenbeck process allows us to embed $T_{\mathcal{O}}$ in a continuous-time flow of transformations on the space $\mathcal{U}$, each of which is $\mathcal{W}_{H_{0}^{1}}$-measure preserving and all but one of which is ergodic. In Section 2, this flow is described via Fourier transforms. In Section 3, a direct and more explicit expression, involving hypergeometric functions and principal values, is computed. In Section 4, we study the two-parameter gaussian process which is induced by the flow introduced in Section 2 , In particular, we compute its covariance and prove that it admits a version which is jointly continuous in its parameters.

\section{Preliminary description of the flow}

Let $\mathcal{O}$ and $T_{\mathcal{O}}$ be the transformations on $H_{0}^{1}$ and $\Theta$ given by (1.1) and (1.2), and recall the unitary map $F: H_{0}^{1} \longrightarrow H^{U}$ in (1.3) and its continuous extension as an isometry from 
$\Theta$ onto $\mathcal{U}$. Clearly, the inverse of $F$ is given by

$$
F^{-1}(\omega)(t)=\sqrt{t} \omega(\log t) \quad \text { for } t>0 .
$$

Because $F$ is unitary and $\mathcal{O}$ is orthogonal on $H_{0}^{1},-F \circ \mathcal{O} \circ F^{-1}$ is an orthognal transformation on $H^{U}$, and because

$$
S:=-F \circ T_{\mathcal{O}} \circ F^{-1}
$$

is continuous extension of $-F \circ \mathcal{O} \circ F^{-1}$ to $\mathcal{U}$, we can identify $S$ as $T_{-F \circ O \circ F^{-1}}$.

Another expression for action of $S$ is

$$
[S(\omega)](t)=-\omega(t)+\int_{0}^{\infty} e^{-\frac{s}{2}} \omega(t-s) d s \quad \text { for } t \in \mathbb{R} .
$$

Equivalently,

$$
S(\omega)=\omega * \mu,
$$

where $\mu$ is the finite, signed measure $\mu$ given by

$$
\mu:=-\delta_{0}+e^{-\frac{t}{2}} \mathbb{1}_{t \geq 0} d t
$$

To confirm that $\omega * \mu$ is well-defined as a Lebesgue integral and that it maps $\mathcal{U}$ continuously into itself, note that, for any $\omega \in \mathcal{U}$ and $t \in \mathbb{R}$,

$$
\begin{aligned}
\int_{0}^{\infty} e^{-\frac{s}{2}}|\omega(t-s)| d s & \leq\|\omega\|_{\mathcal{U}} \int_{0}^{\infty} e^{-\frac{s}{2}} \log (e+|t|+s) d s \\
& \leq\|\omega\|_{\mathcal{U}} \log (e+|t|) \int_{0}^{\infty} e^{-\frac{s}{2}}(1+s) d s \leq 9\|\omega\|_{\mathcal{U}} \log (e+|t|)
\end{aligned}
$$

The Fourier transform $\widehat{\mu}$ of $\mu$ is given by

$\widehat{\mu}(\lambda)=\int_{\mathbb{R}} e^{-i \lambda x} d \mu(x)=-1+\int_{0}^{\infty} e^{-x(1 / 2+i \lambda)} d x=-1+\frac{1}{1 / 2+i \lambda}=\frac{1-2 i \lambda}{1+2 i \lambda}=e^{-2 i \operatorname{Arctg}(2 \lambda)}$.

Hence, for all $h \in H^{U}$ and $\lambda \in \mathbb{R}$,

$$
\widehat{h * \mu}(\lambda)=e^{-2 i \operatorname{Arctg}(2 \lambda)} \widehat{h}(\lambda),
$$

which, since

$$
\|h\|_{H^{U}}^{2}=\frac{1}{8 \pi} \int_{\mathbb{R}}|\widehat{h}(\lambda)|^{2}\left(1+4 \lambda^{2}\right) d \lambda
$$

provides another proof that $S \uparrow H^{U}$ is isometric.

The preceeding, and especially (2.1), suggests a natural way to embed $S \uparrow H^{U}$ into a continuous group of orthogonal transformations. Namely, for $u \in \mathbb{R}$, let $\mu^{* u}$ to be the unique tempered distribution whose Fourier transform is given by

$$
\widehat{\mu^{* u}}(\lambda)=e^{-2 i u \operatorname{Arctg}(2 \lambda)},
$$

and define $\mathcal{S}^{u} \varphi=\varphi * \mu^{* u}$ for $\varphi$ in the Schwartz test function class $\mathscr{S}$ of smooth functions which, together with all their derivatives, are rapidly decreasing. Because

$$
\widehat{\mathcal{S}^{u} \varphi}(\lambda)=e_{4}^{-2 i u \operatorname{Arctg}(2 \lambda)} \hat{\varphi}(\lambda),
$$


it is obvious that $\mathcal{S}^{u}$ has a unique extension as an orthogonal transformation on $H^{U}$, which we will again denote by $\mathcal{S}^{u}$. Furthermore, it is clear that $\mathcal{S}^{u+v}=\mathcal{S}^{u} \circ \mathcal{S}^{v}$ for all $u, v \in \mathbb{R}$. Finally, for all $g, h \in H^{U}, u \in \mathbb{R}$,

$$
\begin{aligned}
\left(g, \mathcal{S}^{u} h\right)_{H^{U}} & =\frac{1}{8 \pi} \int_{\mathbb{R}} \overline{\widehat{g}(\lambda)} \widehat{h}(\lambda) e^{-2 i u \operatorname{Arctg}(2 \lambda)}\left(1+4 \lambda^{2}\right) d \lambda \\
& =\frac{1}{16 \pi} \int_{-\pi / 2}^{\pi / 2} \overline{\widehat{g}\left(\frac{\tan (\tau)}{2}\right)} \widehat{h}\left(\frac{\tan (\tau)}{2}\right)\left(1+\tan ^{2}(\tau)\right)^{2} e^{-2 i u \tau} d \tau,
\end{aligned}
$$

where

$$
\begin{aligned}
& \frac{1}{16 \pi} \int_{-\pi / 2}^{\pi / 2}\left|\widehat{g}\left(\frac{\tan (\tau)}{2}\right)\right|\left|\widehat{h}\left(\frac{\tan (\tau)}{2}\right)\right|\left(1+\tan ^{2}(\tau)\right)^{2} d \tau=\frac{1}{8 \pi} \int_{\mathbb{R}}|\widehat{g}(\lambda)||\widehat{h}(\lambda)|\left(1+4 \lambda^{2}\right) d \lambda \\
& \quad \leq \frac{1}{8 \pi}\left(\int_{\mathbb{R}}|\widehat{g}(\lambda)|^{2}\left(1+4 \lambda^{2}\right) d \lambda\right)^{1 / 2}\left(\int_{\mathbb{R}}|\widehat{h}(\lambda)|^{2}\left(1+4 \lambda^{2}\right) d \lambda\right)^{1 / 2}=\|g\| H_{H^{U}}|| h \|_{H^{U}}<\infty .
\end{aligned}
$$

Hence, by Riemann-Lebesgue lemma, shows that $\left(g, \mathcal{S}^{u} h\right)_{H^{U}}$ tends to zero when $|u|$ goes to infinity.

Now define the associated transformations $S^{u}:=T_{\mathcal{S}^{u}}$ on $\mathcal{U}$ for each $u \in \mathbb{R}$. By the general theory summarized in the introduction and the preceding discussion, we know that $\left\{S^{u}: u \in \mathbb{R}\right\}$ is a flow of $\mathcal{W}_{H^{U}}$-measure preserving transformations and that, for each $u \neq 0$, $S^{u}$ is ergodic.

\section{A MORE EXPLICIT EXPRESSION}

So far we know very little about the transformations $S^{u}$ for general $u \in \mathbb{R}$. By getting a handle on the tempered distributions $\mu^{* u}$, in this section we will attempt to find out a little more.

We begin with the case when $u$ is an integer $n \in \mathbb{Z}$. Recalling that $\mu=-\delta_{0}+e^{-\frac{t}{2}} \mathbb{1}_{t \geq 0} d t$, one can use induction to check that, for $n \geq 0$,

$$
\mu^{* n}=(-1)^{n}\left(\delta_{0}+e^{-\frac{t}{2}} L_{n}^{\prime}(t) \mathbb{1}_{t \geq 0} d t\right),
$$

where $L_{n}$ is the $n$th Laguerre polynomial. Indeed, the Laguerre polynomials satisfy the following relations: for all $n \geq 0$,

$$
L_{n}(0)=1
$$

and for all $n \geq 0, t \in \mathbb{R}$,

$$
L_{n+1}^{\prime}(t)=L_{n}^{\prime}(t)-L_{n}(t) .
$$

Similarly, starting from $\mu^{*-1}=-\delta_{0}+e^{\frac{t}{2}} \mathbb{1}_{t \geq 0} d t$, one finds that

$$
\mu^{* n}=(-1)^{n}\left(\delta_{0}+e^{\frac{t}{2}} L_{n}^{\prime}(-t) \mathbb{1}_{t \leq 0} d t\right)
$$

for $n \leq 0$. In particular, $\mu^{* n}$ is a finite, signed measure for $n \in \mathbb{Z}$ and $S^{n} \omega$ can be identified as $\mu^{* n} * \omega$ for all $\omega \in \mathcal{U}$ and $n \in \mathbb{Z}$.

As the next result shows, when $u \notin \mathbb{Z}, \mu^{* u}$ is more singular tempered distribution than a finite, signed measure.

Proposition 3.1. For each $u \notin \mathbb{Z}$, the distribution $\mu^{* u}$ is given by the following formula:

$$
\mu^{* u}=\cos (\pi u) \delta_{0}(x)+\frac{\sin (\pi u)}{\pi} p v(1 / x)+\Phi_{u}(x),
$$


where $p v$ denotes the principal value, and $\Phi_{u} \in L^{2}(\mathbb{R})$ is the function for which $\Phi_{u}(x)$ equals

$$
\begin{aligned}
e^{-|x| / 2}\left(-\frac{u \sin (\pi u)}{\pi} \sum_{k=0}^{\infty} \frac{(1-u \operatorname{sgn}(x))_{k}|x|^{k}}{k !(k+1) !}[\right. & \frac{\Gamma^{\prime}}{\Gamma}(1+k-u \operatorname{sgn}(x))-\frac{\Gamma^{\prime}}{\Gamma}(1+k) \\
& \left.\left.-\frac{\Gamma^{\prime}}{\Gamma}(2+k)+\log (|x|)\right]+\frac{\sin (\pi u)}{\pi x}\right)-\frac{\sin \pi u}{\pi x},
\end{aligned}
$$

$\Gamma^{\prime} / \Gamma$ being the logarithmic derivative of the Euler gamma function and ()$_{k}$ being the Pochhammer symbol.

Proof. Define the functions $\psi_{u}$ and $\theta_{u}$ from $\mathbb{R}^{*}=\mathbb{R} \backslash\{0\}$ to $\mathbb{R}$ so that $\theta_{u}(x)=e^{-\frac{x}{2}} \psi_{u}(x)$ and $\psi_{u}(x)$ equals

$$
\begin{aligned}
-\frac{u \sin (\pi u)}{\pi} \sum_{k=0}^{\infty} \frac{(1-u \operatorname{sgn}(x))_{k}|x|^{k}}{k !(k+1) !}[ & \frac{\Gamma^{\prime}}{\Gamma}(1+k-u \operatorname{sgn}(x))-\frac{\Gamma^{\prime}}{\Gamma}(1+k) \\
& \left.-\frac{\Gamma^{\prime}}{\Gamma}(2+k)+\log (|x|)\right]+\frac{\sin (\pi u)}{\pi x} .
\end{aligned}
$$

From Lebedev [3], p. 264, equation (9.10.6), with the parameters $\alpha=1-u$ or $\alpha=1+u$, $n=1, z=x$ or $z=-x$, the function $\psi_{u}$ satisfies, for all $x \in \mathbb{R}^{*}$, the differential equation:

$$
x \psi_{u}^{\prime \prime}(x)+(2-|x|) \psi_{u}^{\prime}(x)+(u-\operatorname{sgn}(x)) \psi_{u}(x)=0,
$$

and grows at most polynomially at infinity. One then deduces that $\theta_{u}$ decreases as least exponentially at infinity, and satisfies (for $x \neq 0$ ) the following equation:

$$
x \theta_{u}^{\prime \prime}(x)+2 \theta_{u}^{\prime}(x)+\left(u-\frac{x}{4}\right) \theta_{u}(x)=0 .
$$

At the same time, by writing

$$
e^{-|x| / 2}=\left(e^{-|x| / 2}-1\right)+1
$$

and expanding $\theta_{u}(x)$ accordingly, we obtain:

$$
\begin{aligned}
\theta_{u}(x)= & \frac{\sin (\pi u)}{\pi x}-\frac{u \sin (\pi u)}{\pi}\left[\frac{\Gamma^{\prime}}{\Gamma}(1-u \operatorname{sgn}(x))-\frac{\Gamma^{\prime}}{\Gamma}(1)-\frac{\Gamma^{\prime}}{\Gamma}(2)+\log (|x|)\right] \\
& -\frac{\sin (\pi u)}{2 \pi} \operatorname{sgn}(x)+\eta_{u}(x),
\end{aligned}
$$

for

$$
\eta_{u}(x)=x \eta_{u}^{(1)}(x)+|x| \eta_{u}^{(2)}(x)+x \log (|x|) \eta_{u}^{(3)}(x)+|x| \log (|x|) \eta_{u}^{(4)}(x),
$$

where $\eta_{u}^{(1)}, \eta_{u}^{(2)}, \eta_{u}^{(3)}, \eta_{u}^{(4)}$ are all smooth functions. The derivatives of the functions $x,|x|$, $x \log |x|,|x| \log |x|$ in the sense of the distributions are obtained by interpreting their ordinary derivatives as distributions. Similarly, the product by $x$ of their second distributional derivatives are obtained by multiplying their ordinary second derivatives by $x$. Hence, both $\eta_{u}^{\prime}(x)$ and $x \eta_{u}^{\prime \prime}(x)$ as distributions can be obtained by computing $\eta_{u}^{\prime}(x)$ and $x \eta_{u}^{\prime \prime}(x)$ as functions on $\mathbb{R}^{*}$.

Now, let $\nu_{u}$ be the distribution given by the expression:

$$
\nu_{u}(x)=\cos (\pi u) \delta_{0}(x)+\frac{\sin (\pi u)}{\pi} p v(1 / x)+\left[\theta_{u}(x)-\frac{\sin (\pi u)}{\pi x}\right] .
$$


Note that the term in brackets, in the definition of $\nu_{u}$, is a locally integrable function, and that $\nu_{u}$ coincides with the function $\theta_{u}$ in the complement of the neighborhood of zero. Let us now prove that $\nu_{u}$ satisfies the analog of the equation (3.2), in the sense of the distributions. One has:

$$
\begin{aligned}
\nu_{u}(x)= & \cos (\pi u) \delta_{0}(x)+\frac{\sin (\pi u)}{\pi} p v(1 / x)-\frac{u \sin (\pi u)}{\pi}\left[\frac{\Gamma^{\prime}}{\Gamma}(1-u \operatorname{sgn}(x))\right. \\
& \left.-\frac{\Gamma^{\prime}}{\Gamma}(1)-\frac{\Gamma^{\prime}}{\Gamma}(2)+\log (|x|)\right]-\frac{\sin (\pi u)}{2 \pi} \operatorname{sgn}(x)+\eta_{u}(x) .
\end{aligned}
$$

Since

$$
\frac{\Gamma^{\prime}}{\Gamma}(1+u)-\frac{\Gamma^{\prime}}{\Gamma}(1-u)=\frac{\frac{d}{d u}(\Gamma(1+u) \Gamma(1-u))}{\Gamma(1+u) \Gamma(1-u)}=\frac{\frac{d}{d u}(\pi u / \sin (\pi u))}{\pi u / \sin (\pi u)}=\frac{1}{u}-\pi \cot (\pi u),
$$

one obtains, after straightforward computation,

$\nu_{u}(x)=\cos (\pi) \delta_{0}(x)+\frac{\sin (\pi u)}{\pi} p v(1 / x)-\frac{u \cos (\pi u)}{2} \operatorname{sgn}(x)-\frac{u \sin (\pi u)}{\pi} \log (|x|)+c(u)+\eta_{u}(x)$,

where $c(u)$ does not depend on $x$. One deduces that

$$
\nu_{u}(x)=\cos (\pi u) \delta_{0}(x)+\frac{\sin (\pi u)}{\pi} p v(1 / x)+\chi_{u, 1}(x),
$$

where $\chi_{u, 1}$ denotes a locally integrable function. Moreover,

$$
\nu_{u}^{\prime}(x)=\cos (\pi u) \delta_{0}^{\prime}(x)-\frac{\sin (\pi u)}{\pi} f p\left(1 / x^{2}\right)-u \cos (\pi u) \delta_{0}(x)-\frac{u \sin (\pi u)}{\pi} p v(1 / x)+\eta_{u}^{\prime}(x),
$$

where $f p\left(1 / x^{2}\right)$ denotes the finite part of $1 / x^{2}$, and then

$$
x \nu_{u}^{\prime}(x)=-\cos (\pi u) \delta_{0}(x)-\frac{\sin (\pi u)}{\pi} p v(1 / x)-\frac{u \sin (\pi u)}{\pi}+x \eta_{u}^{\prime}(x) .
$$

By differentiating again, one obtains:

$$
\nu_{u}^{\prime}(x)+x \nu_{u}^{\prime \prime}(x)=-\cos (\pi u) \delta_{0}^{\prime}(x)+\frac{\sin (\pi u)}{\pi} f p\left(1 / x^{2}\right)+\eta_{u}^{\prime}(x)+x \eta_{u}^{\prime \prime}(x) .
$$

Therefore,

$$
\begin{gathered}
x \nu_{u}^{\prime \prime}(x)+2 \nu_{u}^{\prime}(x)+\left(u-\frac{x}{4}\right) \nu_{u}(x)=\chi_{u, 2}(x)+\left(-\cos (\pi u) \delta_{0}^{\prime}(x)+\frac{\sin (\pi u)}{\pi} f p\left(1 / x^{2}\right)\right) \\
+\left(\cos (\pi u) \delta_{0}^{\prime}(x)-\frac{\sin (\pi u)}{\pi} f p\left(1 / x^{2}\right)-u \cos (\pi u) \delta_{0}(x)-\frac{u \sin (\pi u)}{\pi} p v(1 / x)\right) \\
+u\left(\cos (\pi u) \delta_{0}(x)+\frac{\sin (\pi u)}{\pi} p v(1 / x)\right)=\chi_{u, 2}(x)
\end{gathered}
$$

where $\chi_{u, 2}$ is a locally integrable function. Since $\theta_{u}$ satisfies (3.2), $\chi_{u, 2}$ is identically zero. Hence, $\nu_{u}$ is a tempered distribution solving the differential equation:

$$
x \nu_{u}^{\prime \prime}(x)+2 \nu_{u}^{\prime}(x)+\left(u-\frac{x}{4}\right) \nu_{u}(x)=0,
$$

or equivalently,

$$
\frac{x}{4} \nu_{u}(x)-\frac{d^{2}}{d^{2} x}\left(x \nu_{u}(x)\right)-u \nu_{u}(x)=0
$$


Multiplying by $-4 i$ and taking the Fourier transform (in the sense of the distributions), one deduces:

$$
{\widehat{\nu_{u}}}^{\prime}(\lambda)\left(1+4 \lambda^{2}\right)=-4 i u \widehat{\nu_{u}}(\lambda) .
$$

This linear equation admits a unique solution, up to a multiplicative factor $c$ :

$$
\widehat{\nu_{u}}(\lambda)=c \exp \left(\int_{0}^{\lambda} \frac{-4 i u}{1+4 t^{2}} d t\right)=c \exp (-2 i u \operatorname{Arctg}(2 \lambda)) .
$$

Hence, $\nu_{u}$ is proportional to $\mu^{* u}$. In order to determine the constant $c$, let us observe that the distribution $\nu_{u, 0}$ given by

$$
\nu_{u, 0}(x)=\nu_{u}(x)-c \cos (\pi u) \delta_{0}(x)-\frac{c \sin (\pi u)}{\pi} p v(1 / x)
$$

admits the Fourier transform:

$$
\widehat{\nu_{u, 0}}(\lambda)=c e^{-2 i u \operatorname{Arctg}(2 \lambda)}-c e^{-\pi i u \operatorname{sgn}(\lambda)}
$$

One deduces that $\widehat{\nu_{u, 0}}$ is a function in $L^{2}$, which implies that $\nu_{u, 0}$ is also a function in $L^{2}$, and then locally integrable. Since the last term in (3.3) is also a locally integrable function, one deduces that $c=1$, and then

$$
\mu^{* u}=\nu_{u}
$$

which proves Proposition 3.1.

The reasonably explicit expression for $\mu^{* u}$ found in Proposition 3.1 yields a reaonably explicit expression for the action of $\mathcal{S}^{u}$. Indeed, only the term $p v(1 / x)$ is a source of concern. However, convolution with respect of $p v(1 / x)$ is, apart from a multiplicative constant, just the Hilbert transform, whose properties are well-known. In particular, it is a translation invariant, bounded map on $L^{2}(\mathbb{R})$, and as such it is also a bounded map on $H^{U}$. Thus, we can unambiguously write $\mathcal{S}^{u}(h)=h * \mu^{* u}$ for all $h \in H^{U}$. On the other hand, the interpretation of $\omega * \mu^{* u}$ for $\omega \in \mathcal{U}$ needs some thought. No doubt, $\omega * \mu^{* u}$ is well-defined as an element of $\mathscr{S}^{\prime}$, the space tempered distributions, but it is not immediately obvious that it is can be represented by an element of $\mathcal{U}$ or, if it can, that the element of $\mathcal{U}$ which represents it can be identified as $S^{u} \omega$. In fact, the best that we should expect is that such statements will be true of $\mathcal{W}_{H^{U}}$-almost every $\omega \in \mathcal{U}$. The following result justifies that expectation.

Proposition 3.2. For $\mathcal{W}_{H^{U}}$-almost every $\omega \in \mathcal{U}$, the tempered distribution $\omega * \mu^{* u}$ is represented by an element of $\mathcal{U}$ which can be can be identified as $S^{u} \omega$.

Proof. Recall that, for $\varphi \in \mathscr{S}, \varphi * \mu^{*-u}$ is the element of $\mathscr{S}$ whose Fourier transform is given by

$$
\widehat{\varphi * \mu^{*-u}}(\lambda)=\widehat{\varphi}(\lambda) e^{2 i u \operatorname{Arctg}(2 \lambda)} \quad \text { for all } \lambda \in \mathbb{R} .
$$

Also, if $T \in \mathscr{S}^{\prime}$, then $T * \mu^{* u}$ is the tempered distribution whose action on $\varphi \in \mathscr{S}$ is given by

$$
\mathscr{S}\left\langle\varphi, T * \mu^{* u}\right\rangle_{\mathscr{S}^{\prime}}=\mathscr{S}\left\langle\varphi * \mu^{*-u}, T\right\rangle_{\mathscr{S}^{\prime}}
$$

Now choose an orthonormal basis $\left\{h_{n}: n \geq 1\right\}$ for $H^{U}$ all of whose members are elements of $\mathscr{S}$, and, for each $n \geq 1$, set $g_{n}=\frac{1}{4} h_{n}+h_{n}^{\prime \prime}$. Next, think of $g_{n}$ as the element of $\mathcal{U}^{*}$ whose action on $\omega \in \mathcal{U}$ is given by

$$
\mathcal{U}\left\langle\omega, g_{n}\right\rangle_{\mathcal{U}^{*}}=_{8}\left\langle g_{n}, \omega\right\rangle_{\mathscr{S}^{\prime}}
$$


It is then an easy matter to check that, in the notation of the introduction, $h_{n}=h_{g_{n}}$. Hence, if $B$ is the subset of $\omega \in \mathcal{U}$ for which

$$
\omega=\lim _{n \rightarrow \infty} \sum_{m=1}^{n} \mathscr{S}\left\langle g_{n}, \omega\right\rangle_{\mathscr{S}^{\prime}} h_{n} \quad \text { and } \quad S^{u} \omega=\lim _{n \rightarrow \infty} \sum_{m=1}^{n} \mathscr{S}\left\langle g_{n}, \omega\right\rangle_{\mathscr{S}^{\prime}} h_{n} * \mu^{* u},
$$

where the convergence is in $\mathcal{U}$, then $\mathcal{W}_{H^{U}}(B)=1$.

Now let $\omega \in B$. Then, for each $\varphi \in \mathscr{S}$,

$$
\begin{aligned}
\mathscr{S}\left\langle\varphi, \omega * \mu^{* u}\right\rangle_{\mathscr{S}^{\prime}} & =\mathscr{S}\left\langle\varphi * \mu^{*-u}, \omega\right\rangle_{\mathscr{S}^{\prime}}=\lim _{n \rightarrow \infty} \sum_{m=1}^{n} \mathscr{S}\left\langle g_{n}, \omega\right\rangle_{\mathscr{S}^{\prime} \mathscr{S}}\left\langle\varphi, h_{n} * \mu^{* u}\right\rangle_{\mathscr{S}^{\prime}} \\
& =\lim _{n \rightarrow \infty} \sum_{m=1}^{n} \mathscr{S}\left\langle g_{n}, \omega\right\rangle_{\mathscr{S}^{\prime} \mathscr{S}}\left\langle\varphi, \mathcal{S}^{u} h_{n}\right\rangle_{\mathscr{S}^{\prime}}=\mathscr{S}\left\langle\varphi, S^{u} \omega\right\rangle_{\mathscr{S}^{\prime}} .
\end{aligned}
$$

Thus, for $\omega \in B, \omega * \mu^{* u} \in \mathscr{S}^{\prime}$ is represented by $S^{u} \omega \in \mathcal{U}$.

\section{A TWO PARAMETER GAUSSIAN PROCESS}

By construction, $\left\{S^{u} \omega(t):(u, t) \in \mathbb{R}^{2}\right\}$ is a gaussian family in $L^{2}\left(\mathcal{W}_{H^{U}}\right)$. In this concluding section, we will show that this family admits a modification which is jointly continuous in $(u, t)$.

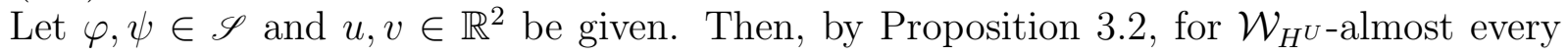
$\omega \in \mathcal{U}$,

$$
\iint_{\mathbb{R}^{2}} \varphi(s) \psi(t)\left(S^{u}(\omega)\right)(s)\left(S^{v}(\omega)\right)(t) d s d t=\mathscr{S}\left\langle\varphi, \omega * \mu^{* u}\right\rangle_{\mathscr{S}^{\prime} \mathscr{S}}\left\langle\psi, \omega * \mu^{* v}\right\rangle_{\mathscr{S}^{\prime}}
$$

where the integral in the left-hand side is absolutely convergent. Because $\mathbb{E}_{\mathcal{W}_{H^{U}}}\left[S^{u} \omega(t)^{2}\right]$ is finite and independent of $(u, t) \in \mathbb{R}^{2}$, by taking the expectation with respect to $\mathcal{W}_{H^{U}}$ and using (2.2), one can pass from this to

$$
\begin{aligned}
& \iint_{\mathbb{R}^{2}} \varphi(s) \psi(t) \mathbb{E}_{\mathcal{W}_{H^{U}}}\left[\left(S^{u}(\omega)\right)(s)\left(S^{v}(\omega)\right)(t)\right] d s d t=\mathbb{E}_{\mathcal{W}_{H}}\left[\mathscr{S}\left\langle\varphi, \omega * \mu^{* u}\right\rangle_{\mathscr{S}^{\prime} \mathscr{S}}\left\langle\psi, \omega * \mu^{* v}\right\rangle_{\mathscr{S}^{\prime}}\right] \\
& =\frac{2}{\pi} \int_{-\infty}^{\infty} \frac{e^{2 i(u-v) \operatorname{Arctg}(2 \lambda)}}{1+4 \lambda^{2}} \widehat{\varphi}(\lambda) \overline{\widehat{\psi}(\lambda)} d \lambda=\frac{2}{\pi} \iiint_{\mathbb{R}^{3}} \frac{e^{i[(t-s) \lambda+2(u-v) \operatorname{Arctg}(2 \lambda)]}}{1+4 \lambda^{2}} \varphi(s) \psi(t) d s d t d \lambda .
\end{aligned}
$$

Hence,

$$
\left.\mathbb{E}_{\mathcal{W}_{H U}}\left[S^{u}(\omega)\right)(s)\left(S^{v}(\omega)\right)(t)\right]=\frac{2}{\pi} \int_{-\infty}^{\infty} \frac{e^{i[(t-s) \lambda+2(u-v) \operatorname{Arctg}(2 \lambda)]}}{1+4 \lambda^{2}} d \lambda
$$

first for almost every and then, by continuity, for all $(s, t) \in \mathbb{R}^{2}$. In particular, we now know

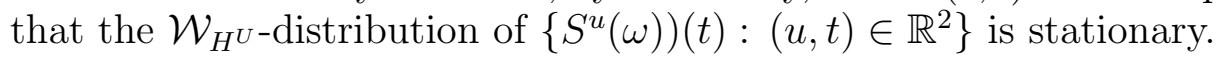

To show that there is a continuous version of this process, we will use Kolmogorov's continuity criterion, which, because it is stationary and gaussian, comes down to showing that

$$
\left|1-\mathbb{E}_{\mathcal{W}_{H^{U}}}\left[\left(S^{u}(\omega)\right)(s)\left(S^{v}(\omega)\right)(t)\right]\right| \leq C|(u, s)-(v, t)|^{\alpha}
$$


for some $C<\infty$ and $\alpha>0$. But

$$
\begin{aligned}
& \left|1-\mathbb{E}_{\mathcal{W}_{H^{U}}}\left[\left(S^{u}(\omega)\right)(s)\left(S^{v}(\omega)\right)(t)\right]\right| \leq \frac{2}{\pi} \int_{-\infty}^{\infty} \frac{d \lambda}{1+4 \lambda^{2}}\left|e^{i[(t-s) \lambda+2(u-v) \operatorname{Arctg}(2 \lambda)]}-1\right| \\
& \leq \frac{2}{\pi} \int_{-\infty}^{\infty} \frac{d \lambda}{1+4 \lambda^{2}}\left|e^{i(t-s) \lambda}-1\right|+\frac{2}{\pi} \int_{-\infty}^{\infty} \frac{d \lambda}{1+4 \lambda^{2}}\left|e^{2 i(u-v) \operatorname{Arctg}(2 \lambda)}-1\right| \\
& \leq \frac{2}{\pi} \int_{-\infty}^{\infty} \frac{d \lambda}{1+4 \lambda^{2}}(|t-s||\lambda| \wedge 2)+\frac{4}{\pi} \int_{-\infty}^{\infty} \frac{d \lambda}{1+4 \lambda^{2}}|(u-v) \operatorname{Arctg}(2 \lambda)|,
\end{aligned}
$$

and, after simple estimation, this shows that

$$
\left|1-\mathbb{E}\left[\left(S^{u}(\omega)\right)(s)\left(S^{v}(\omega)\right)(t)\right]\right| \leq C\left[|u-v|+|t-s|\left(1+\log \left(1+\frac{1}{(t-s)^{2}}\right)\right)\right],
$$

where $C<\infty$. Clearly, the desired conclusion follows.

Remark 4.1. A question about filtrations comes naturally when one considers the group of transformations $\left(S^{u}\right)_{u \in \mathbb{R}}$ on the space $\mathcal{U}$. Indeed, for all $t, u \in \mathbb{R}$, let $\mathcal{F}_{t}^{u}$ be the $\sigma$-algebra generated by the $\mathcal{W}_{H^{U}}$-negligible subsets of $\mathcal{U}$ of and the variables $\left(S^{u}(\omega)\right)(s)$, for $s \in(-\infty, t]$ (these variables are well-defined up to a negligible set). From the results of Jeulin and Yor, one quite easily deduces the following properties of the filtrations of the form $\left(\mathcal{F}_{t}^{u}\right)_{t \in \mathbb{R}}$ for $u \in \mathbb{R}:$

- For all $t, u \in \mathbb{R}, \mathcal{F}_{t}^{u}$ is generated by $\mathcal{F}_{t}^{u+1}$ and $\left(S^{u}(\omega)\right)(t)$.

- For all $t, u \in \mathbb{R}, \mathcal{F}_{t}^{u+1}$ and $\left(S^{u}(\omega)\right)(t)$ are independent under $\mathcal{W}_{H^{U}}$.

- For all $t, u \in \mathbb{R}$, the decreasing intersection of $\mathcal{F}_{t}^{u+n}$ for $n \in \mathbb{Z}$ is trivial (i.e. it satisfies the zero-one law).

- If $u \in \mathbb{R}$ is fixed, the $\sigma$-algebra generated by $\mathcal{F}_{t}^{u+n}$ for $t \in \mathbb{R}$ does not depend on $n \in \mathbb{Z}$.

All these statements concern the sequence of filtrations $\left(\mathcal{F}^{u+n}\right)_{n \in \mathbb{Z}}$ for fixed $u \in \mathbb{R}$. A natural question arises: how can these results be extended to the continuous family of filtrations $\left(\mathcal{F}^{u}\right)_{u \in \mathbb{R}}$ ? Unfortunately, for the moment, we have no answer to this question (in particular the family does not seem to be decreasing with $u$ ).

\section{REFERENCES}

[1] T. Jeulin, M. Yor, Filtration des ponts browniens et équations différentielles stochastiques linéaires, Séminaire de Probabilités, XXIV, Lecture Notes in Math., vol. 1426, Springer-Verlag, 1990, pp. 227265.

[2] P.-A. Meyer, Sur une transformation du mouvement brownien due à Jeulin et Yor, Séminaire de Probabilités, XXVIII, Lecture Notes in Math., vol. 1583, Springer-Verlag, 1994, pp. 98-101.

[3] N.-N. Lebedev, Special functions and their applications (Translated from Russian), Dover, New York, 1972.

[4] M. Yor, Some aspects of Brownian motion. Part I: Some Special Functionals, Lectures in Mathematics, Ed. ETH Zürich, Birkhaüser, 1992.

[5] D. Stroock, Probability Theory, an Analytic View, 2nd edition, Cambridge University Press, 2011.

[6] D. Stroock, Some thoughts about Segal's ergodic theorem, Colloq. Math. vol. 118 \#1, pp. 89-105, 2010. 